\title{
4 Plataforma estadística de variables + atmosféricas con la aplicación de las normas TULSMA en el cantón Cuenca
}

Cabrera Lituma, Alexandraํ ; Sellers Walden, Chester²

Ingeniera de Sistemas y Telemática por la Universidad del Azuay e investigadora en el IERSE de la Universidad del Azuay, en el área de la distribución, publicación de variables atmosféricas en la ciudad de Cuenca, y el fortalecimiento de la Infraestructura de Datos Espaciales.

alecabreralituma@gmail.com

Ingeniero de Sistemas por la Universidad del Azuay, Master Universitario en Gestión Sostenible de la Tierra y Territorio por la Universidad Santiago de Compostela, Magister en Geomática con mención en Ordenamiento Territorial por la Universidad del Azuay. Docente e Investigador en el IERSE de la Universidad del Azuay.

csellers@uazuay.edu.ec

\section{Resumen}

La contaminación del aire afecta a la salud de los seres humanos, por lo que es fundamental conocer su estado y presentarlo a la ciudadanía. Se necesita de una herramienta de gestión ambiental para la toma de decisiones en beneficio de la comunidad. Este proyecto presenta una plataforma web para el análisis estadístico de variables atmosféricas registradas en la estación de monitoreo automático de la

\section{Alexandra Cabrera Lituma ${ }^{1}$}

\section{Chester Sellers Walden ${ }^{2}$}

EMOV-EP. El estudio incluye el nivel de la calidad del aire, tablas y gráficos estadísticos de este comportamiento, los mismos que se basan en la normas del Texto Unificado de Legislación Ambiental Secundaria (TULSMA).

\section{Palabras clave:}

Calidad del aire, contaminación atmosférica, TULSMA, plataforma web, variables meteorológicas. 


\begin{abstract}
Air pollution affects the health of human beings so it is essential to know its status and present it to the public. An environmental management tool is needed to make decisions for the benefit of the community. This project presents a web platform for the statistical analysis of atmospheric variables recorded in EMOVEP's automatic monitoring station. The
\end{abstract}

study includes air quality level, tables and statistical graphs of this behavior, which are based on the regulations of the Unified Text of Secondary Environmental Legislation (TULSMA).

\section{Keywords:}

Air quality, air pollution, TULSMA, web platform, meteorological variables.

\section{Introducción}

El aumento de la circulación vehicular en el centro de la ciudad de Cuenca genera emisiones de gases que producen problemas en la calidad de vida de los ciudadanos. Esto afecta a su salud, por lo que es necesario dar a conocer la situación en la que se desenvuelven.

La Empresa Pública Municipal de Movilidad, Tránsito y Transporte de Cuenca (EMOV-EP) a través de su red de monitoreo trabaja conjuntamente con la Universidad del Azuay, a través del Instituto de Estudios de Régimen Seccional del Ecuador- IERSE en la publicación de las variables atmosféricas recogidas por medio de su estación de monitoreo automático, con el fin de proporcionar a los ciudadanos información del estado de contaminación atmosférica, en tiempo real.

\section{Marco teórico}

\subsection{Contaminación atmosférica}

La contaminación atmosférica es causada por las alteraciones que sufre el aire debido a la presencia de sustancias en el ambiente que producen daños temporales o permanentes en los seres vivos. Entre las principales fuentes que provocan la mayor contaminación al entorno tenemos a las industrias y los automotores que generan óxido de nitrógeno $(\mathrm{V})$, azufre (S) y Dióxido de carbono (CO_2) (United States Environmental Protection Agency , 2016).

\subsection{Contaminantes Atmosféricos}

\subsubsection{Ozono $\left(\mathrm{O}_{3}\right)$}

Es un gas muy reactivo, incoloro, de olor fuerte, compuesto por 3 átomos de oxígeno formado al exponerse a la radiación solar.

Cuando la concentración de ozono es mayor a $180 \mathrm{~g} / \mathrm{m}^{3}$ es perjudicial para la salud porque provoca tos, náuseas, dolor de cabeza, dificultades al respirar. Este se manifiesta en la época de calor (Zuk, Tzintzum, \& Rojas, 2007).

\subsubsection{Dióxido de nitrógeno $\left(\mathrm{NO}_{2}\right)$}

Es un gas de color café, compuesto por la presencia de óxido de nitrógeno, que se forma en los cilindros de los motores, emitido en su mayoría por los vehículos, industrias y plantas de energía (Zuk, Tzintzum, \& Rojas, 2007). 
La presencia de dióxido de nitrógeno afecta al sistema respiratorio principalmente de los niños y de las personas asmáticas. La exposición a corto plazo en niveles altos provoca deterioros en las células pulmonares, mientras que a largo plazo en niveles bajos causa daños al tejido pulmonar (Sellers, 2017).

\subsubsection{Monóxido de carbono (CO)}

Es un gas incoloro e inodoro. El 96\% es de origen antropogénico generado por la combustión incompleta de petróleo, gas, gasolina y tabaco. Un $70 \%$ se debe a las emisiones en los vehículos y el humo del tabaco. Este afecta a los pulmones, corazón y cerebro debido a la reducción de oxígeno en la sangre (Puigcerver \& Carrascal, 2008).

\subsubsection{Partículas finas $\left(\mathrm{PM}_{2.5}\right)$}

También conocidas como partículas respirables, se forman por las emisiones de una fuente antropogénica como los vehículos y aparatos eléctricos domésticos y de forma natural cuando ocurren incendios o erupciones volcánicas. Están asociadas con enfermedades respiratorias como asma, disfunciones pulmonares y cardiovasculares (Zuk, Tzintzum, \& Rojas, 2007).

\subsubsection{Dióxido de azufre $\left(\mathrm{SO}_{2}\right)$}

Gas incoloro, no inflamable, se presenta natural por la actividad volcánica y artificial en los procesos de combustión del petróleo, quema del carbón e industrias metalúrgicas (The International Volcanic Health Hazard Network, 2005).

El $\mathrm{SO}_{2}$ en altas concentraciones origina problemas para respirar, irritación crónica en las vías respiratorias lo que debilita las defensas, provocando bronquitis y bronco espasmos, problemas respiratorios y car- diovasculares presente en la mayoría de casos en niños y personas adultas (Sellers, 2017).

\subsection{Variables meteorológicas}

Son fenómenos producidos a corto plazo en las capas bajas de la atmósfera, donde desenvuelven las actividades antrópicas y naturales de los seres vivos (Sierra, 2006).

\subsubsection{Presión barométrica}

Se entiende a la presión barométrica como el peso del aire, que ejerce una fuerza sobre un cuerpo debido a la gravedad (Rodríguez, Capa, \& Portela, 2004).

La combinación de una alta presión atmosférica, baja temperatura y alta humedad puede llevar a problemas en el sistema circulatorio provocando problemas a la salud, como una hemorragia intracerebral. (Diez, 2006)

\subsubsection{Humedad relativa}

Está representada por la cantidad de vapor de agua que contiene al aire; esa cantidad no es fija ya que se debe a distintos factores como el nivel del mar, la precipitación, cobertura vegetal, etcétera.

Si la humedad relativa se encuentra por debajo del $20 \%$ puede causar problemas en la salud ya que la deshidratación de las membranas del tracto respiratorio origina infecciones bacterianas (Rodríguez, Capa, \& Portela, 2004).

\subsubsection{Precipitación}

Una nube está compuesta por millones de gotas de agua y cristales de hielo, originarios del cambio de estado de vapor del agua en una masa de aire, que al tener 
contacto con la atmósfera fría produce la saturación. La cantidad e intensidad de precipitación depende del contenido y velocidad de la humedad en el aire (Maderey \& Jiménez, 2005).

La precipitación que llega a la superficie terrestre se da en distintas formas como Iluvia, nieve, granizo y neblina (Sierra, 2006).

Una cantidad elevada de precipitación conjunta con bajas temperaturas y fuertes vientos puede afectar a la salud provocando problemas cerebrovasculares, ataques cardiacos, estrés físico y mental (Diez, 2006).

\subsubsection{Radiación solar}

La radiación solar se comprende como la energía trasladada del Sol a la atmósfera terrestre, la cual viaja por el espacio en forma de ondas llevando una cantidad de energía (Rodríguez, Capa, \& Portela, 2004).

La exposición a la radiación solar en exceso provoca problemas a la piel como quemaduras, arrugas, cáncer de piel, entre otros. Las quemaduras se producen debido a la exposición a los rayos ultravioletas lo que provoca que la piel se vuelva roja, inflamada; aparezcan ampollas, conjuntamente con descamaciones. Esto se presenta normalmente después de 24 horas de exposición. (Cecofar, 2014).

\subsubsection{Velocidad y dirección del viento}

El viento se debe al movimiento del aire de un lugar a otro provocado debido a la diferencia de presión o temperatura. (Rodríguez, Capa, \& Portela, 2004).

Si la velocidad llega a $1 \mathrm{~m} / \mathrm{s}$ no es sentido por las personas, si se encuentra entre
1 y $4 \mathrm{~m} / \mathrm{s}$ se siente ligeramente, pero si es superior a los $8 \mathrm{~m} / \mathrm{s}$ se siente con fuerza (Diez, 2006).

Cuando se asocia el viento fuerte con una temperatura baja provoca dolores en las articulaciones en las personas reumáticas, baja autoestima lo que produce depresión y trastornos emocionales (Gutiérrez, 2007).

\subsection{Normas aplicadas}

\subsubsection{Legislación ecuatoriana}

En la ley de prevención y control de la contaminación ambiental del Ecuador, Registro Oficial suplemento N418 en el artículo 1 expresa "Queda prohibido expeler hacia la atmósfera o descargar en ella, sin sujetarse a las correspondientes normas técnicas y regulaciones, contaminantes que, a juicio de los Ministerios de Salud y del Ambiente, en sus respectivas áreas de competencias, puedan perjudicar la salud y vida humana, la flora, la fauna y los recursos o bienes del Estado o de particulares o constituir una molestia" (Sistema Integrado de Legislación Ecuatoriana, 2004).

\subsubsection{Texto Unificado de Legislación Secundaria del Ministerio del Ambiente (TULSMA)}

En la reforma del libro VI del TULSMA de la calidad del aire emitido por el Ministerio del Ambiente del Ecuador MAE, el 4 de mayo del 2015 se establecen los siguientes principios que serán de apoyo para el caso de estudio.

\subsubsection{Preventivo o de prevención}

"Es la obligación que tiene el Estado, a través de sus instituciones y órganos y de 
acuerdo a las potestades públicas asignadas por ley, de adoptar las políticas y medidas oportunas que eviten los impactos ambientales negativos, cuando exista certidumbre de daño." (Ministerio del Ambiente, 2015)

\subsubsection{Precautorio o de precaución}

"Es la obligación que tiene el Estado, a través de sus instituciones y órganos y de acuerdo a las potestades públicas asignadas por ley, de adoptar medidas protectoras eficaces y oportunas cuando haya peligro de daño grave o irreversible al ambiente, aunque haya duda sobre el impacto ambiental de alguna acción, u omisión o no exista evidencia científica del daño.

El principio de precaución se aplica cuando es necesario tomar una decisión u optar entre alternativas en una situación en que la información técnica y científica es insuficiente o existe un nivel significativo de duda en las conclusiones del análisis técnico-científico. En tales casos el principio de precaución requiere que se tome la deci- sión que tiene el mínimo riesgo de causar, directa o indirectamente, daño al ecosistema." (Ministerio del Ambiente, 2015)

\subsubsection{Corrección en la fuente}

"Es la obligación de los sujetos de control de adoptar todas las medidas pertinentes para evitar, minimizar, mitigar y corregir los impactos ambientales desde el origen del proceso productivo. Este principio se aplicará en los proyectos y en adición a planes de manejo o de cualquier naturaleza previstos en este Libro." (Ministerio del Ambiente, 2015)

\subsection{Estación de monitoreo automático}

La EMOV-EP cuenta con una estación de monitoreo automático en tiempo real de variables atmosféricas, se encuentra localizada en la parte superior de la Alcaldía de Cuenca, ubicada en la calle Bolívar 7-67 y Borrero. Esta estación automática tiene un rango de cobertura de aproximadamente $4 \mathrm{~km}$, con lo0 que se cubre la mayor parte de Cuenca.

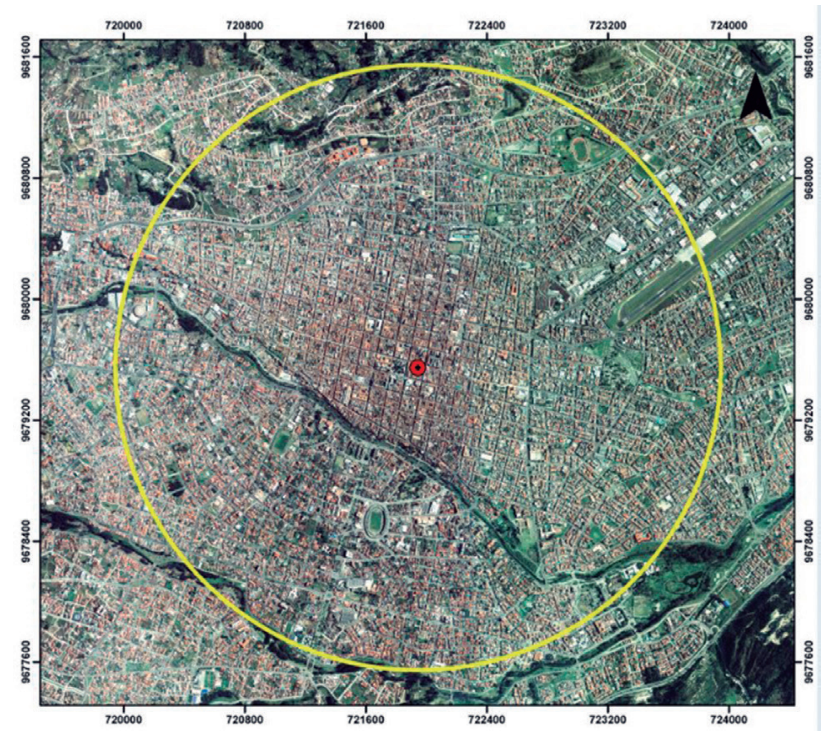

Figura 1. Área de cobertura estación automática 


\section{Metodología}

\subsection{Captura, almacenamiento y sistematización de las variables atmosféricas}

La captura de los datos se realiza a través del datalogger, cada segundo, información que es almacenada en la base de datos de la EMOV-EP, por la seguridad de la infraestructura de los registros de esta, la cual crea una base de datos espejo, sincronizando la información cada 20 minutos establecida por tareas programadas.

Para la comunicación se estableció un canal por medio de una VPN Check Point Endpoint Security y un programa aplicativo, el cual transfiere los datos espejo de la EMOV-EP al servidor de la UDA cada 20 minutos con información de cada minuto.

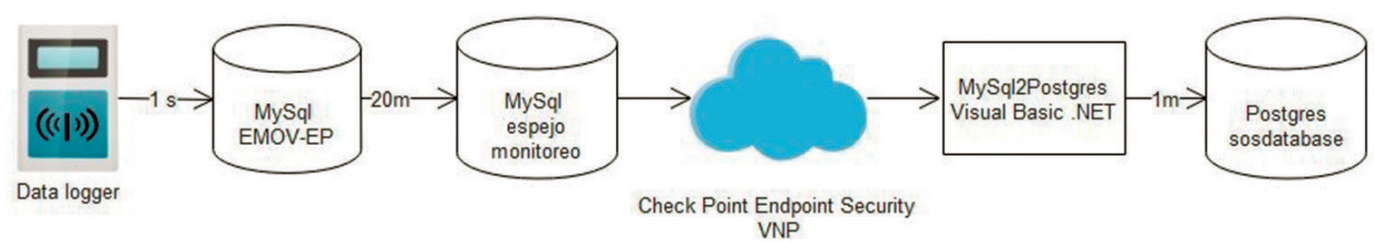

Figura 2. Esquema transferencia de datos

\subsection{Transformación y} estandarización de los datos

Los datos de las variables atmosféricas capturadas en la estación de monitoreo automático de la EMOV-EP pasan por un filtro en el que se eliminan los registros erróneos debidos a calibración de los sensores o a fallas eléctricas. Además los valores son corregidos de acuerdo a las condiciones locales, tomando en cuenta la temperatura y la presión barométrica del lugar en donde se tomó el dato. Los registros están tomados en las unidades de medida de la tabla siguiente. 
Tabla 1. Unidades de medida

\begin{tabular}{|c|c|}
\hline $\begin{array}{c}\text { Variable } \\
\text { atmosférica }\end{array}$ & Descripción \\
\hline $\mathrm{CO}$ & $\mathrm{mg} / \mathrm{m}^{3}$ \\
\hline $\mathrm{O}_{3}$ & $\mathrm{ug} / \mathrm{m}^{3}$ \\
$\mathrm{NO}_{2}$ & $\mathrm{ug} / \mathrm{m}^{3}$ \\
$\mathrm{PM}_{2.5}$ & $\mathrm{ug} / \mathrm{m}^{3}$ \\
$\mathrm{SO}_{2}$ & $\mathrm{ug} / \mathrm{m}^{3}$ \\
\hline
\end{tabular}

\subsubsection{La legislación TULSMA}

La legislación TULSMA establece que los datos se tomen en la unidad de medida de microgramos por medio cúbico $\left(\mathrm{ug} / \mathrm{m}^{3}\right)$. Para el contaminante CO que se encuentra en $\mathrm{mg} / \mathrm{m}^{3}$ se multiplica por 1000 para convertir a ug $/ \mathrm{m}^{3}$. Además los valores se redondean a tres decimales.

En la calidad del aire se toma el valor promedio generado en el periodo de tiem- po establecido por la norma de los contaminantes con la aplicación de la tabla 2.

La calidad del aire puede ubicarse en los niveles de bueno, alerta, alarma y emergencia. La figura 3 indica los valores mínimos que deben tener para estar dentro del nivel. En el caso en que el valor de calidad del aire sea menor al valor de alerta se considera como bueno.

\begin{tabular}{|c|c|c|c|}
\hline $\begin{array}{c}\text { CONTAMINANTE Y PERIODO DE } \\
\text { TIEMPO }\end{array}$ & ALERTA & ALARMA & EMERGENCIA \\
\hline $\begin{array}{c}\text { Monóxido de carbono } \\
\text { Concentración promedio en ocho horas } \\
\left(\mu \mathrm{g} / \mathrm{m}^{3}\right)\end{array}$ & 15000 & 30000 & 40000 \\
\hline $\begin{array}{c}\text { Ozono } \\
\text { Concentración promedio en ocho horas } \\
\left(\mu \mathrm{g} / \mathrm{m}^{3}\right)\end{array}$ & 200 & 400 & 600 \\
\hline $\begin{array}{c}\text { Dióxido de Nitrógeno } \\
\text { Concentración promedio en una hora }\left(\mu \mathrm{g} / \mathrm{m}^{3}\right)\end{array}$ & 1000 & 2000 & 3000 \\
\hline $\begin{array}{c}\text { Dióxido de azufre } \\
\text { Concentración promedio en veinticuatro horas } \\
\left(\mu \mathrm{g} / \mathrm{m}^{3}\right)\end{array}$ & 200 & 1000 & 1800 \\
\hline $\begin{array}{c}\text { Material particulado PM 10 } \\
\text { Concentración en veinticuatro horas }\left(\mu \mathrm{g} / \mathrm{m}^{3}\right)\end{array}$ & 250 & 400 & 500 \\
\hline $\begin{array}{c}\text { Material particulado PM } \mathbf{2 , 5} \\
\text { Concentración en veinticuatro horas }\left(\mu \mathrm{g} / \mathrm{m}^{3}\right)\end{array}$ & 150 & 250 & 350 \\
\hline
\end{tabular}

Figura 3. Criterios de concentraciones TULSMA (Ministerio del Ambiente, 2015) 
Después de determinar la calidad del aire de cada uno de los contaminantes se establece el criterio de nivel y el color de acuerdo con la Tabla 2.

Tabla 2. Criterios de nivel TULSMA

\section{Calidad de aire}

\begin{tabular}{|c|c|}
\hline Color simbolizado & Criterio de nivel \\
\hline Verde & Bueno \\
\hline Amarillo & Alerta \\
\hline Naranja & Alarma \\
\hline Marrón & Emergencia \\
\hline
\end{tabular}

\section{Resultados}

El principal resultado de la elaboración e implementación de este proyecto es una plataforma web para el monitoreo y publicación de los contaminantes atmosféricos y de las variables meteorológicas de la ciudad de Cuenca para cumplir con la legislación TULSMA. El sitio web se encuentra en el geoportal de la UDA en el siguiente vínculo: http://gis.uazuay.edu.ec/ide2015/ sistematulsma.php
A primera vista se observa la concentración de los contaminantes atmosféricos, el periodo de tiempo y el rango en el que estos se encuentran.

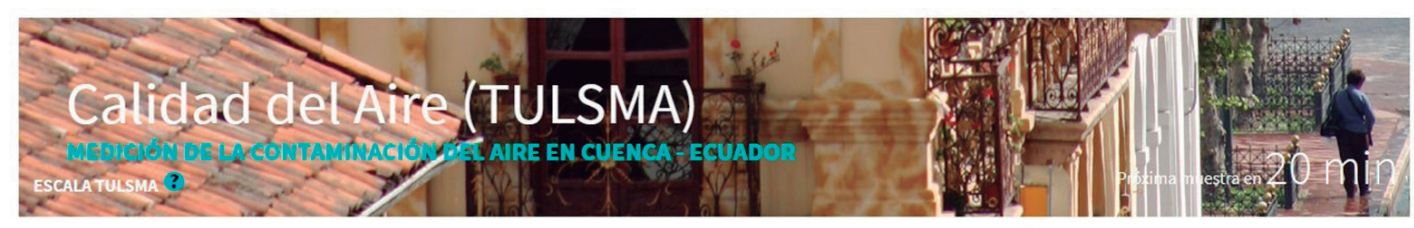

\section{Concentraciones de los contamiantes}

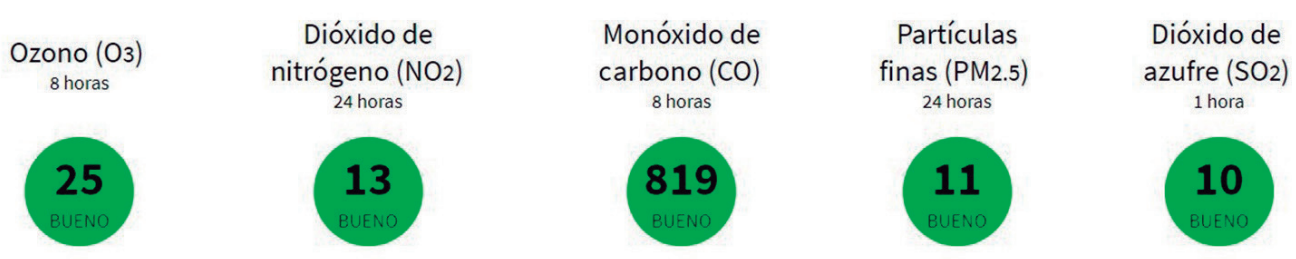

Figura 4. Concentraciones contaminantes 
También presenta la generación del gráfico lineal de variables atmosféricas en periodos de tiempo de un día. La herramienta está desarrollada para permitir la selección de los distintos contaminantes y un rango horario con el cual se construirá el gráfico respectivo.

\section{Gráfica de los contaminantes} Período mostrado:
DESDE: 2017-6-13 00:00 $\triangleright \mathrm{O} 3 \unlhd \mathrm{SO} 2 \square \mathrm{PM} 2 \_5 \square \mathrm{CO} \square \mathrm{NO} 2$

HASTA: 2017-6-13 23:59

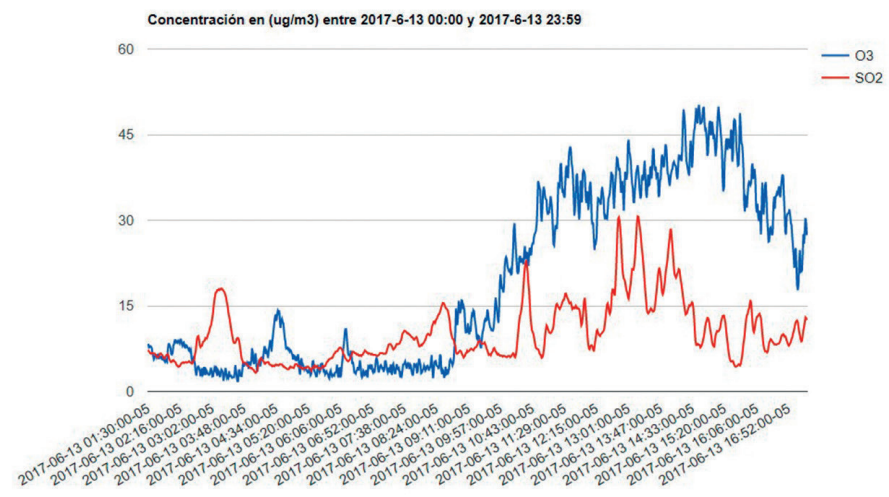

Figura 5. Gráfico de los contaminantes atmosféricos

Las mediciones estadísticas se muestran en dos formatos: El primero de estadística básica por medio de una tabla que contiene la unidad, desviación estándar, varianza y moda por contaminante.

\section{Estadísticas: Básicas}

\begin{tabular}{|c|c|c|c|c|c|}
\hline \multicolumn{6}{|c|}{ Registros encontrados:2 } \\
\hline No. & Contaminante & Unidad & DE & Varianza & Moda \\
\hline 1 & $\mathrm{O} 3$ & $\mathrm{ug} / \mathrm{m} 3$ & 15.531 & 241.212 & 38.011 \\
\hline 2 & $\mathrm{SO} 2$ & $\mathrm{ug} / \mathrm{m} 3$ & 5.189 & 26.93 & 6.096 \\
\hline
\end{tabular}

Figura 6. Datos estadísticas básicas 
El segundo, de estadística general a través un gráfico de barras que representa los valores de la tabla de datos de estadística general, y contiene la unidad de medida, los mínimos, promedios y máximos por contaminante. Las mediciones de los valores estadísticos se obtienen dependiendo del contaminante y el periodo de tiempo establecido por el usuario.

\section{Estadística: General}

Registros encontrados:2

No. Contaminante Unidad Mínimo Promedio Maximo

$\begin{array}{cccccc}1 & \mathrm{SO} 2 & \mathrm{ug} / \mathrm{m} 3 & 3.273 & 10.02 & 30.684 \\ 2 & \mathrm{O} 3 & \mathrm{ug} / \mathrm{m} 3 & 1.775 & 19.593 & 50.224\end{array}$

Gráfica estadística: General

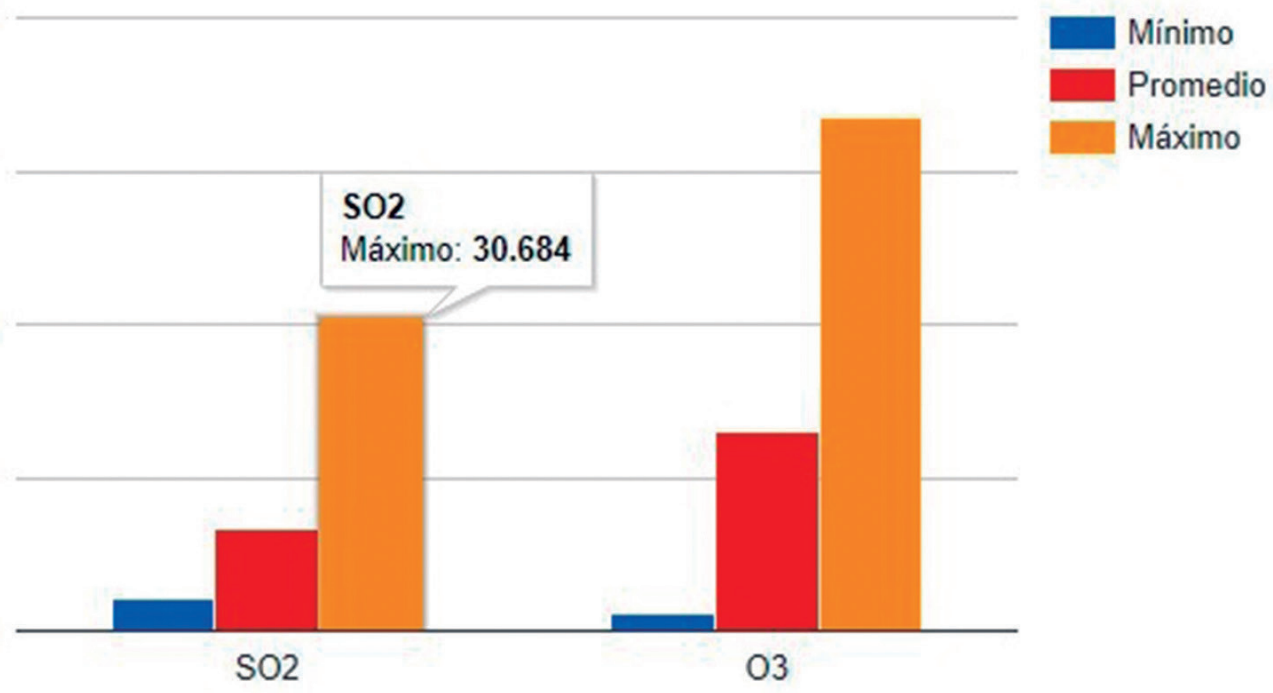

Figura 7. Representación estadística general 
Con respecto a la disponibilidad de los rangos de tiempo se implementó la herramienta que facilita al usuario personalizar su consulta, estableciendo el rango de tiempo de acuerdo con sus necesidades lo que permite visualizar los datos históricos. Esto ayuda a tener una visión clara de cómo se van relacionando los comportamientos de las variables atmosféricas dependiendo del cambio climático, incendios, tráfico vehicular, entre otros factores.

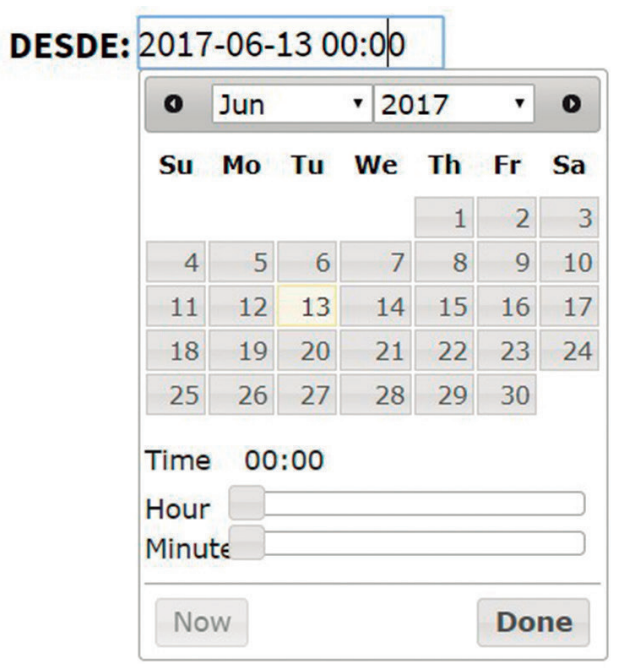

\section{HASTA: 2017-06-13 23:59}

\begin{tabular}{|c|c|c|c|c|c|}
\hline - Jun & & . 20 & & $\checkmark$ & 0 \\
\hline Su Mo & Tu & We & Th & $\mathrm{Fr}$ & Sa \\
\hline & & & 1 & 2 & 3 \\
\hline 4 & 6 & 7 & 8 & 9 & 10 \\
\hline \begin{tabular}{l|l}
11 & 12 \\
\end{tabular} & 13 & 14 & 15 & 16 & 17 \\
\hline \begin{tabular}{|l|l|}
18 & 19 \\
\end{tabular} & 20 & 21 & 22 & 23 & 24 \\
\hline \begin{tabular}{l|l}
25 & 26 \\
\end{tabular} & 27 & 28 & 29 & 30 & \\
\hline Time 2 & $: 59$ & & & & \\
\hline Hour & & & & & \\
\hline Minute $\square$ & & & & & \\
\hline Now & & & & Do & ne \\
\hline
\end{tabular}

Figura 8. Ejemplo rango de periodo de tiempo

Conjuntamente se representan las variables meteorológicas de la ciudad de Cuenca por medio de una tabla para cada variable, la cual contiene la unidad de medida, los valores mínimos, promedios y máximos registrados, exceptuando la precipitación que contiene la unidad de medida y el total que es la suma total de todas las precipitaciones. 


\section{Meteorología de la ciudad de Cuenca}

Grados Centigrados

Temperatura del aire

Unidad Mínimo Promedio Máximo

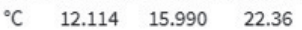

\section{Presión barométrica}

Unidad Mínimo Promedio Máximo

$\begin{array}{llll}\mathrm{hPa} & 749.07 & 752.881 & 758.63\end{array}$

\section{Humedad relativa}

Unidad Mínimo Promedio Máximo

$\begin{array}{llll}\% & 40.877 & 67.957 & 93.238\end{array}$

\section{Precipitación}

Unidad Total

$\mathrm{mm} \quad 7.6$
Radiación solar

Unidad Mínimo Promedio Máximo

W/m2 $\quad 49.367 \quad 384.700 \quad 1214.674$

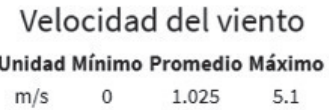

Dirección del viento

Unidad Mínimo Promedio Máximo

$\begin{array}{llll}\circ & 0 & 159.958 \quad 370\end{array}$

Figura 9. Meteorología de la ciudad de Cuenca

\section{Conclusiones}

El proyecto permite a los expertos y a la ciudadanía cuencana contar con una plataforma digital para la consulta del comportamiento de las variables atmosféricas registradas por la estación de monitoreo automático de la EMOV-EP, utilizando las normas del Texto Unificado de Legislación Ambiental Secundaria (TULSMA) del Ecuador.

El usuario accede, en tiempo real, a los reportes estadísticos de las variables por medio de tablas, de gráficos y la presentación de la calidad del aire.

Para la presentación de los datos se dio prioridad a que estos sean comprensibles para la ciudadanía. A través de escalas de colores y mensajes intuitivos se transforma el valor numérico en un conjunto de recomendaciones.

Esta plataforma es una herramienta para la gestión ambiental y la toma de medidas de precaución y prevención del cuidado de la salud de los ciudadanos, además de servir como base para futuros proyectos encaminados a conocer la calidad del aire y el comportamiento de las variables atmosféricas. 


\section{Referencias}

Cecofar. (18 de 06 de 2014). Centro Cooperativo Farmacéutico. Obtenido de Centro Cooperativo Farmacéutico: http:// blog.cecofar.es/enfermedadesradiacion-solar/

Diez, E. C. (2006). Variables meteorológicas y salud. Madrid: Longares I.R, S.A.

EMOV-EP. (2014). Red de Monitoreo EMOV-EP.

EMOV-EP. (2015). Informe de Calidad Aire. Cuenca.

Gutiérrez, R. (12 de 2007). Zona hospitalaria. Obtenido de Zona hospitalaria: http://www.zonahospitalaria.com/elfrio-y-los-huesos/

Maderey, L., \& Jiménez, A. (2005). Principios de Hidrogeofrafía Estudio del Ciclo Hidrológico. México: Universidad Nacional Autónoma de México.

Ministerio del Ambiente. (04 de 05 de 2015). Ministerio del Ambiente. Obtenido de Ministerio del Ambiente: http://suia.ambiente.gob.ec/ documents/10179/185880/ACUERD O+061+REFORMA+LIBRO+VI+TULS MA+-+R.0.316+04+DE+MAYO+2015. pdf/3c02e9cb-0074-4fb0-afbe$0626370 f a 108$

Ministerio del Ambiente. (2015). Norma de calidad del aire ambiente o nivel de inmisión. En M. d. Ambiente, Texto unificado de legislación secundaria del ministerio del ambiente.

Puigcerver, M., \& Carrascal, D. (2008). El medio atmosférico: meteorología y contaminación. Barcelona: Universidad de Barcelona.

Rodríguez, R., Capa, Á., \& Portela, A. (2004). Meteorología y Climatología. Fundación Española para la Ciencia y la Tecnología.
Sellers, C., Espinoza, C. (2017). Publicación de los contaminantes atmosféricos de la estación de monitoreo en tiempo real de la ciudad de Cuenca, utilizando servicios OGC. ACl Avances en Ciencias e Ingenierías, Quito.

Sierra, M. M. (2006). Establecer la asociación existente entre las variables meteorológicas, temperatura, velocidad del viento y precipitación y las concentraciones de Pm10 registradas en la red de calidad del aire de Bogotá D.C. Bogotá: Universidad de la Salle.

Sistema Integrado de Legislación Ecuatoriana. (2004). Ley de prevención y control de la contaminación ambiental, Registro Oficial suplemento \# 418.

The International Volcanic Health Hazard Network. (2005). The International Volcanic Health Hazard Network. Obtenido de The International Volcanic Health Hazard Network: http:// ivhhn.org/index.php?option=com_ content\&view=article\&id=144

United States Environmental Protection Agency . (23 de 02 de 2016). United States Environmental Protection Agency. Obtenido de United States Environmental Protection Agency: https://www3.epa.gov/ climatechange/science/causes.html

United States Environmental Protection Agency. (2016). United States Environmental Protection Agency. Obtenido de United States Environmental Protection Agency: https://www3.epa.gov/

Zuk, M., Tzintzum, G., \& Rojas, L. (2007). Tercer almanaque de datos y tendencias de la calidad del aire de nueve ciudades mexicanas. México D.F. 